\title{
RADIATION INSTABILITY IN A STRATIFIED SHEAR FLOW
}

\author{
L. A. Ostrovsky, Yu. A. Stepanyants and L. Sh. Tsimring \\ Institute of Applied Physics, Academy of Sciences of the U.S.S.R., Gorky, U.S.S.R.
}

(Received 3 May 1982; received for publication 10 August 1983)

\begin{abstract}
The paper deals with a new mechanism of instability in stratified fluid caused by internal wave radiation from a shear layer. This radiation instability may be explained in terms of the negativeenergy wave concept. These waves are able to grow due to radiation losses. The dispersion relation and non-linear stage of the process are analyzed through a model with tangential velocity discontinuity. The non-linear description is based on a long-wave approximation leading to an evolution equation of the Korteweg-de Vries type with certain additional terms responsible for instability as well as dissipation due to turbulence. Approximate solutions of the equation are obtained which, in particular, describe the evolution of solitons including explosive growth and approaching the steady state.
\end{abstract}

\section{INTRODUCTION}

Shear instability of a stratified flow is one of the most complicated but fascinating problems in modern hydrodynamics. General stability conditions derived by Miles and Howard were followed by a sequence of special solutions describing the Kelvin-Helmholtz (KH) instability in layers with tangential discontinuities and analogous instability in some simple continuous profiles having a critical layer where the flow velocity coincides with the phase speed of waves. These results are reviewed, for example, in [1-3].

It should be noted, however, that such phenomena have been generally considered within the frames of the linear boundary value problem for an inviscid fluid. Any deviation from these restrictions essentially complicates the task. A general discussion of these problems can be found in the paper by Benjamin [4] who, in particular, introduced the concept of negativeenergy waves (NEW) whose excitation reduces (in a given reference system) the total energy of the "wave and flow" system. It is clear, that introduction of any energy sink for such a wave (dissipation, coupling with another wave with positive energy) results in an increase of the absolute value of this energy, i.e. in instability. Generally speaking, the magnitude and even the sign of the wave energy may depend on the frame of reference, but the condition of stability or instability should be, of course, invariant. Nevertheless, the NEW concept turns to be very useful for understanding the physical situation. In particular, knowing the dispersion characteristics of waves in an ideal fluid one can predict the behavior of the system with the addition of dissipation. Note that such problems are of certain practical interest. For example, in the ocean a pycnocline (i.e. the region of sharp density gradients), as well as elements of fine structure are often at the same time regions of a relatively strong shear flow (see also the end of the paper).

These problems have been studied in detail in electrodynamics [5]. In hydrodynamics NEW have attracted interest only recently, about fifteen years after Benjamin's paper [4]. Some specific models of stratified flows have been considered in which the waves growing due to instability of the KH type coexist with "potentially unstable" NEW. As was shown by Benjamin [4] and later Cairns [6] NEW exist even in the classical Kelvin-Helmholtz model which corresponds to tangential discontinuity separating two fluids of different density.

Taking into account that the energy of a weak wave is $W=\omega I$, where $\omega$ is the frequency and $I$ is the adiabatic invariant (equal to $\partial L / \partial \omega$, with $L$ being the averaged Langragian), one can readily determine the sign of the energy by the dispersion characteristics. In particular, the function $W(\omega, k)$, where $k$ is the wave number, changes the sign at the points where $\omega(k)$ changes its sign or the group velocity goes into infinity $\left(\right.$ since $\left.I \sim\left(\frac{\mathrm{d} \omega}{\mathrm{d} k}\right)^{-1}[7]\right)$.

In terms of physics the basic new factor introduced by stratification is the appearance of the waves (internal waves in the inhomogeneous fluid and surface waves at the water-air interface). These waves, which exist in the absence of a shear flow as well, are capable of 
absorbing the energy from such a flow. Generally speaking, this is a complication, in comparison with a homogeneous flow, but in some important cases the presence of waves makes the problem essentially simpler. For example, a non-linear stage of instability can be analysed taking advantage of the fact that the parameter characterizing the wave-flow energy exchange is small. This is especially true of NEW for which instability is often "weaker" than the $\mathrm{KH}$ instability where the wave usually grows and breaks during the time comparable to its period (this is particularly characteristic of tangential discontinuity in a homogeneous fluid). As a result, asymptotic methods based on small non-linearity approximation are often useful for studying NEW. Voronovich and Rybak [7] and Craik and Adam [8] noted that non-linear "explosive" instability of a wave triplet is possible, that occurs if one of the waves has negative energy. Ostrovsky and Stepanyants [9] considered the growth of finiteamplitude NEW due to viscous dissipation in a two-layer fluid of finite depth. In this case long-wa ve approximation is valid and the problem includes non-linear growth of solitons or cnoidal waves.

We consider here a simple model of a homogeneous finite-thickness liquid layer slipping over infinite stratified liquid. Thus, the interface is a tangential discontinuity and, at the same time, a density discontinuity. In such a model bulk internal waves with frequencies less than the Brunt-Väisälä frequency, may propagate downward. This provides growth of NEW propagating along the interface in the long-wave range, which would remain stable in the frames of a usual Kelvin-Helmholtz model (when the lower liquid is homogeneous).

This "radiation instability" (RI) has been briefly discussed, for the first time, by Ostrovsky and Tsimring [10] in a linear formulation for a slightly different model (when both layers were of infinite depth). Herc we consider both linear and non-linear stages of radiation instability for the model with a finite upper layer. It is important that in this model long waves are practically non-dispersive and have a finite propagation velocity. As a consequence, the NEW lie in a longwave region, as it is seen from the linear analysis performed in Sections 2 and 3, where dispersion characteristics and increments are found for infinitesimal perturbations.

Since the energy losses result in growth of NEW, determination of the factors stabilizing radiation instability may be a rather nontrivial problem. One of these factors, turbulence of the upper moving layer, is considered in Section 4.

The existence of long-wave instability makes it possible to construct, in Section 5, a nonlinear theory of such instability based on derivation of an evolution equation. The latter is a generalization of the Korteweg-de Vries equation containing the terms responsible for growth and decay of the waves. When these terms are sufficiently small one can find approximate solutions of this equation in the form of solitons with slowly varying parameters.

Section 6 gives the summary of the main results, as well as some estimates demonstrating the possibility of radiation instability appearing in the upper ocean layer. This layer is usually characterized by a sharp pycnocline at a finite depth and smooth stratification below the pycnocline and may be roughly approximated by the model considered here.

\section{NEGATIVE-ENERGY WAVES IN A TWO-LAYER MODEL}

To illustrate the mechanism of instability, consider first a fluid layer of a finite thickness $h$ and density $\rho_{i}$ moving at a speed $U$. The upper boundary of the layer $(z=0)$ is supposed to be rigid and the lower one $(z=h)$ is contiguous to the immobile infinitely deep fluid with a density $\rho_{2}>\rho_{1}$ (Fig. 1). Linearizing the basic equations of an ideal fluid and searching for a solution in the form $f(z) \exp [i(k x-\omega t)]$ (where $z$ and $x$ are the vertical and the horizontal coordinates, respectively) we obtain a dispersion equation

$$
\omega_{1,2}=\frac{\rho_{1} U k \pm \sqrt{\left[\left(\rho_{2}-\rho_{1}\right) g k\left(\rho_{1}+\rho_{2} t h k h\right)-\rho_{1} \rho_{2} U^{2} k^{2}\right] t h k h}}{\rho_{1}+\rho_{2} t h k h} .
$$

Here the signs " \pm " refer to two different wave modes. Real parts of $\omega_{1.2}$ are shown in Fig. 2 for two different values of velocity $U$. At $k h \rightarrow \infty$ one can easily obtain a well-known dispersion equation describing waves in a $\mathrm{KH}$ model for an infinite fluid [11]. As follows 


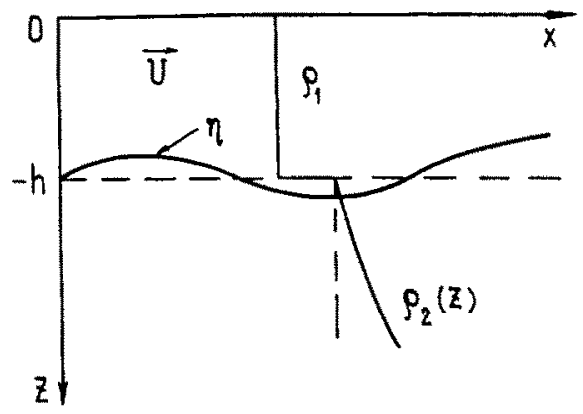

Fig. 1.

from (1), sufficiently short waves (provided that surface tension at the layer interface is neglected) grow at any flow velocity $U$ due to the Kelvin-Helmholtz mechanism. The minimum wave number of the growing waves $k_{\mathrm{KH}}$ depends on velocity $U$ and may be determined from

$$
\frac{\rho_{1}+\rho_{2} t h k_{\mathrm{KH}} h}{k_{\mathrm{KH}} h}=\frac{\rho_{1} \rho_{2}}{\rho_{2}-\rho_{1}} \cdot \frac{U^{2}}{g h} .
$$

Thus, $\mathrm{KH}$ instability does not occur for long-wave perturbations. However, in the stability range $k<k_{\mathrm{KH}}$ the NEW correspond to the shaded parts of the curves in Fig. 2. These parts lie within the range $k_{c} \leq K \leq K_{\mathrm{KH}}$ and in accordance with the previous discussion $\omega_{2}\left(k_{c}\right)=0$, that is on the lower branch of the dispersion curve the frequency changes its sign at the point $k_{c}$, while at $k_{\mathrm{KH}}$ the adiabatic invariant changes its sign $\left(\frac{\mathrm{d} \omega_{2}\left(k_{\mathrm{KH}}\right)}{\mathrm{d} k} \rightarrow \infty\right)$. The first condition gives an equation for $k_{c}$ :

$$
\frac{t h k_{c} h}{k_{c} h}=\frac{\rho_{1}}{\rho_{2}-\rho_{1}} \cdot \frac{U^{2}}{g h}
$$

If $U \geq U_{c}=\sqrt{\frac{\rho_{2}-\rho_{1}}{\rho_{1}}} g h$, NEW occupy all the region of low wave numbers from $k=0$ to $k=k_{\mathrm{KH}}$ (see Fig. 2).

The presence of NEW in this idealized system does not yet mean the existence of instability there. However, these waves may grow if there is an energy sink due to internal wave radiation into a continuously stratified fluid.

\section{RADIATION INSTABILITY}

Now, take into account continuous stratification in the lower fluid characterized by a constant Brunt-Väisälä frequency $N=\sqrt{-\frac{g}{\rho} \frac{\mathrm{d} \rho}{\mathrm{d} z}}$. In addition to the wave adjacent to the

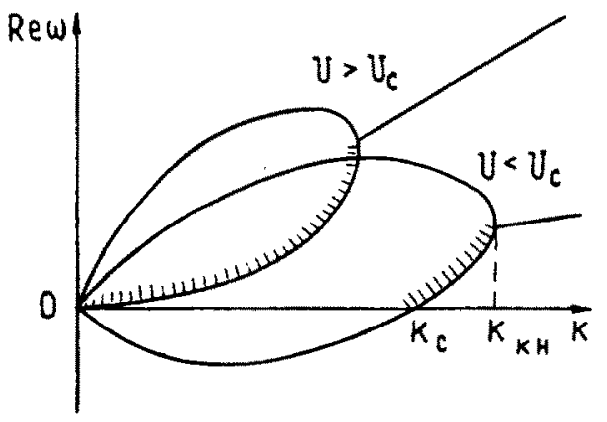

Fig. 2. Dispersion curves of the Kelvin-Helmholtz model at two values of relative layer velocity. The dashed parts of the curves correspond to negative-energy waves. 
layer interface (with $|(\omega)|>N$ ) there are three-dimensional internal waves with $|(\omega)|<N$ propagating below the discontinuity layer. In the lower fluid positive-energy waves may exist in the form $\sim \exp [i(k x+m z-\omega t)]$, where $m^{2}=k^{2}\left(\frac{N^{2}}{\omega^{2}}-1\right)$, the sign of the real part $m$ being determined by the condition of the wave energy radiation from discontinuity (see also $[10]$ ). As a result we obtain a dispersion equation

$$
a(\omega-U k)^{2} \operatorname{coth} k h+\omega \sqrt{\omega^{2}-N^{2}}-(1-a) g k=0,
$$

where $a=\rho_{1} / \rho_{2}$. Figure 3 shows real and imaginary parts as a function of $k$. As seen from the plot, the instability associated with NEW appears in the finite range of $k$ axis. The left instability boundary is determined from the condition $\omega\left(k_{c}\right)=0$ (if $U \geq U_{c}, k_{c}=0$, as in Fig. 3 ) and the right one - from $\omega\left(k_{M}\right)=N$. Generally, there is a stability interval $k_{M} \leq k \leq k_{\mathrm{kH}}$,

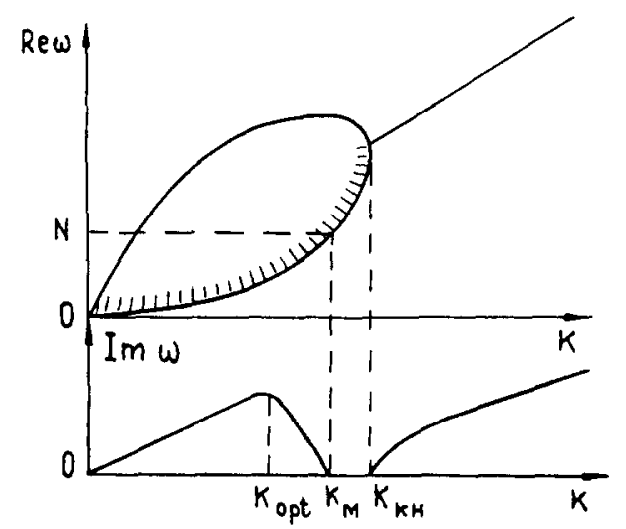

Fig. 3. Real and imaginary parts of frequency as a function of the wave number for the Kelvin-Helmholiz model with a stratified bottom layer.

where $\omega>N$ so that the internal waves cannot propagate in the lower medium. One can obtain from (4) an expression for the increment, i.e. the imaginary part of the wave frequency $\omega_{i}$ assuming it to be small compared to the real part $\omega_{r}$ (this condition is fulfilled if $N \ll(1-a) g / U)$ :

$$
\omega_{i}=-\omega_{r} \frac{\sqrt{N^{2}-\omega_{r}^{2}}}{2 a\left(\omega_{r}-U k\right)} t h k h .
$$

Henceforth we take particular interest in the long-wave approximation $(k h \rightarrow 0)$ similar to the shallow water approximation for surface waves. Within this limit for the lower branch of the dispersion curve $\omega_{r}$ one obtains from (4)

$$
\omega_{r} \simeq \Re+\psi k^{3}
$$

where

$$
\begin{aligned}
& \vartheta=U_{c}(c-1)+i \frac{N h}{2 a}(c-1), \\
& \psi=U_{c} h^{2}\left[\frac{1}{6}+\frac{(c-1)^{2}}{2}+\frac{(c-1)^{3}}{4}\right]-i \frac{U_{c}^{2} h}{N}(c-1)^{3}, \\
& c=\frac{U}{U_{c}} .
\end{aligned}
$$

As seen from (6) and (7), stratification at $z<-h$ results in growth of NEW with small $k$, if $c>1$. Besides, the first dispersive term in the expansion of $\omega_{r}$ is now proportional to $k^{3}$ 
instead of $k^{2}$ for $N=0$ [as it is easy to see from (1)]. Note, that in the range with a growing negative-energy mode there is another positive-energy mode which corresponds to the upper branch of the dispersion curve $\omega_{r}(k)$. This mode does not at all satisfy the conditions of radiation and finiteness at $z \rightarrow-\infty$ and should be described as a damping nonlocalized structure known as a leaky wave [10].

\section{EFFECT OF TURBULENCE IN THE UPPER LAYER}

In many cases phenomena associated with the presence of viscosity and especially turbulent mixing are important. The effect of the upper layer turbulence in a stationary fluid model as applied to oceanic internal waves has been considered by Ostrovsky and Soustova [12]. They have shown that internal wave damping is governed by two basic mechanisms. One of them is associated with turbulent viscosity, the other - with the internal wave energy consumption for variation of turbulent energy in the presence of the buoyant force. Corresponding wave decrements were obtained from a linearized system of semiempirical equations for averaged motions and energy of turbulent pulsations. $\uparrow$ These results can be easily generalized for the case of the moving upper layer considered here. Moreover, for long waves $(k h \rightarrow 0)$ the motion of the upper layer does not change the decrement considerably. Almost all the energy of such waves is concentrated in the upper homogeneous layer [12] whose motion is, therefore, equivalent to the motion of the medium as a whole. In this case the decrement of both internal wave modes due to the upper layer turbulence is

$$
\Gamma(k)=-\frac{p}{g l_{z}(1-a)}-\delta_{T} k^{2},
$$

where $p$ is the turbulent buoyancy flux, $l_{z}$ is the vertical scale of turbulence, $\delta_{T}$ is the turbulent viscosity coefficient. Note, that both the terms in (8) yield damping of positive and negative energy waves. (If, however, the lower fluid were turbulized, long NEW could be amplified due to losses even without account of radiation; cf. [9].) The first term in (8) does not depend on $k$, therefore the turbulent diffusion mechanism is of particular importance for long internal waves.

With simultaneous account of turbulence in the upper layer and stratification in the lower one, waves corresponding to the lower branch of the dispersion curve (Fig. 3) grow in some range of $k$ and decrease beyond it. The dependence of the overall decrement $\gamma=\omega_{i}+\Gamma$ on $k$ at small $k$ can be roughly approximated by a cubic parabola

$$
\gamma(k)=-\varepsilon+\mu k-\delta_{T} k^{2}-v k^{3} .
$$

The coefficients in the expression are defined by

$$
\varepsilon=\frac{p}{g l_{z}(1-a)} ; \mu=\frac{N h}{2 a}(c-1) ; v=\frac{U_{c}^{2} h}{2 N}(c-1)^{3} .
$$

Figure 4 shows $\gamma$ as a function of $k$ as calculated from Eqs (5) and (8) (broken line) and from

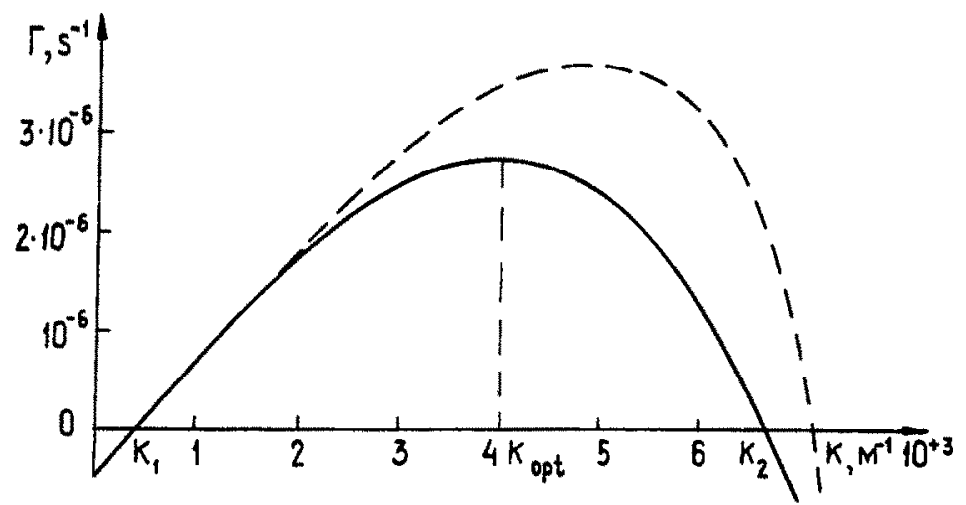

Fig. 4. Growth rate as a function of the wave number at $p=5 \times 10^{-9} \mathrm{~m}^{2} \mathrm{~s}^{-3} ; 1=1 \mathrm{~m}$; $a=p_{1} / \rho_{2}=0.999 ; N=5 \times 10^{-4} \mathrm{~s}^{-1} ; h=50 \mathrm{~m} ; c=U / U_{\mathrm{c}}=1,1 ; \delta_{T}=10^{2} \mathrm{~m}^{2} \mathrm{~s}^{-1}$.

$\dagger$ Weak $I W$ damping when the decrement is small compared to the frequency, is considered here. If the turbulence is more intense or the $I W$ length is small, turbulence effect on the mode structure should be taken into account [13]. 
the approximate formula (9) (solid curve). The maximum increment $\gamma_{\max }$ and the corresponding wave number $k_{\text {opt }}$ are roughly defined by

$$
\gamma_{\max }=\frac{N^{2} h}{\sqrt{6} U_{c}}-\frac{\delta_{T} N^{2}}{3 U_{c}^{2}(c-1)^{2}}-\varepsilon ; \quad k_{\mathrm{opt}}=\frac{N}{\sqrt{3} U_{c}(c-1)} .
$$

Figure 5 shows $\gamma_{\max }$ and $k_{\text {opt }}$ as well as the limits $k_{1}$ and $k_{2}$ of the instability range as a function of the upper layer thickness $h$.

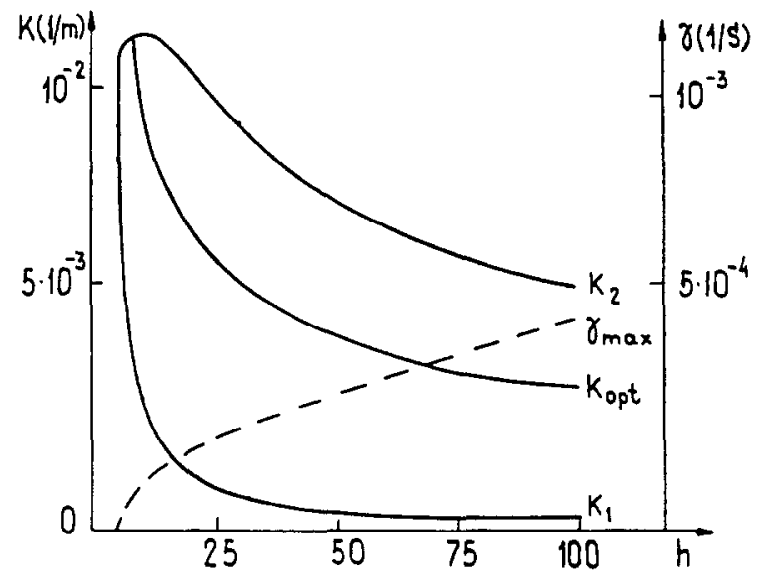

Fig. 5. $k_{1}, k_{2}, k_{\text {opl }}$, and $\gamma_{\max }$ (broken line) as a function of the upper layer thickness. Parameters are the same as in Fig. 4.

Thus, the analysis of the linear model approximation shows that at $c>1$ there is an internal wave energy source in the range $k_{1} \leq k \leq k_{2}$ that is stipulated both by the shear flow and stratification of the lower fluid. Adjacent to this range on both sides are regions of wave damping due to the upper layer turbulence.

\section{LONG NON-LINEAR WAVES}

We now consider the behavior of non-linear perturbations including the possibility of stationary wave formation. An equation describing propagation of weakly non-linear internal waves can be derived from general hydrodynamic equations by means of an asymptotic technique (see, for example, [14]). The linear terms in this equation can be obtained by inverse Fourier transformation of the dispersion equation (6) multiplied by the unknown function $\eta(x, t)$. In this case $\omega$ should be changed for $i \frac{\partial \eta}{\partial t}$; in the real part of $(6) k$ is changed for $-i \frac{\partial \eta}{\partial x}\left(k^{3} \rightarrow i \frac{\partial^{3} \eta}{\partial x^{3}}\right)$ and in the imaginary part $k$ and $k^{3}$ are changed for the Gilbert transformation of $\frac{\partial \eta}{\partial x}$ and $\frac{\partial^{3} \eta}{\partial x^{3}}$, respectively (cf. [15]). The non-linear term can be derived disregarding the terms responsible for dispersion and amplification of the wave [14]. In this way we obtain a generalized Korteweg-de Vries (KdV) equation with a number of additional nonconservative terms in the RHS which describe wave amplification and dissipation

$$
\begin{gathered}
\frac{\partial \eta}{\partial t}+V \frac{\partial \eta}{\partial x}+\alpha \eta \frac{\partial \eta}{\partial x}-\beta \frac{\partial^{3} \eta}{\partial x^{3}}=\hat{S}(\eta), \\
\hat{S}(\eta) \equiv-\varepsilon \eta+\frac{\mu}{\pi} \oint_{-\infty}^{\infty} \frac{\partial \eta}{\partial x^{\prime}} \frac{\mathrm{d} x^{\prime}}{x-x^{\prime}}+\delta_{T} \frac{\partial^{2} \eta}{\partial x^{2}}+\frac{v}{\pi} \oint_{-\infty}^{\infty} \frac{\partial^{3} \eta}{\partial x^{\prime 3}} \frac{\mathrm{d} x^{\prime}}{x-x^{\prime}} .
\end{gathered}
$$

Here $\eta(x, t)$ is the deviation of the interface from its equilibrium position $V=\operatorname{Re} \vartheta, \beta=\operatorname{Re} \psi$, $\alpha=\frac{3}{2} \frac{U_{c}}{h}$ is the non-linearity coefficient; $f_{-\infty}^{\infty}$ represents as usual the principal value of the 
integral. The second term in the RHS of (12) is similar to that describing a simple model of Landau damping for ion-acoustic waves in plasma $[15,16]$, but here its sign corresponds to the wave amplification. If $\hat{S}(\eta)$ is small compared to the non-linear and dispersive terms in the LHS of (12), one can construct approximate quasi-stationary solutions according to the following scheme. In the limit $\hat{S}(\eta)=0 \mathrm{Eq}$. (12) transforms to a $\mathrm{KdV}$ equation which has well-known periodic and solitary solutions. If $\hat{S}(\eta)$ is nonzero but small enough, there exist solutions close to the corresponding stationary ones for the $\mathrm{KdV}$ equation, but their parameters (amplitude, period etc.) are slowly varying functions of time (see, for example, [14]). Time dependence of these parameters can be determined from the energy balance equation which follows from (12) after multiplying it by $\eta$ and integrating over $x$ (this is a well-known procedure, see, for example, $[14,16])$ :

$$
\begin{aligned}
\frac{1}{2} \frac{\mathrm{d}}{\mathrm{d} t}\left\langle\eta^{2}\right\rangle= & -\varepsilon\left\langle\eta^{2}\right\rangle+\frac{\mu}{\pi}\left\langle\eta \oint_{-\infty}^{\infty} \frac{\partial \eta}{\partial x^{\prime}} \frac{\mathrm{d} x^{\prime}}{x-x^{\prime}}\right\rangle \\
& -\delta_{r}\left\langle\left(\frac{\partial \eta}{\partial x}\right)^{2}\right\rangle-\frac{v}{\pi}\left\langle\eta f_{-\infty}^{\infty} \frac{\partial^{3} \eta}{\partial x^{\prime 3}} \frac{\mathrm{d} x^{\prime}}{x-x^{\prime}}\right\rangle,
\end{aligned}
$$

where $\langle\cdots\rangle=\frac{1}{\lambda} \int_{0}^{\lambda} \ldots \mathrm{d} x$ for a periodic wave with a wavelength $\lambda$ and $\langle\cdots\rangle=\int_{-\infty}^{\infty} \ldots \mathrm{d} x$ for a soliton. First consider solution of Eq. (10) close to a stationary cnoidal wave

$$
\eta(x, t)=\frac{12 \beta k^{2}}{\alpha \pi} K(q) \frac{\mathrm{d}}{\mathrm{d} \theta} z_{n}\left[\frac{K(q)}{\pi} \theta, q\right],
$$

where $q$ is the squared modulus of elliptic functions which is here the non-linearity parameter related to the wave amplitude by

$$
A=\frac{12 \beta k^{2}}{\alpha \pi^{2}} q K(q)
$$

( $A$ is the difference between the maximum and the minimum values of $\eta$ ), $\theta=\omega t-k\left(x-V_{s} t\right), \omega=\frac{4 \beta k^{3}}{\pi^{2}} K^{2}(q)\left[2-q-3 \frac{E(q)}{K(q)}\right], z_{n}$ is the Jacobi zeta function with a period $2 \pi$ with respect to 0 and a zero mean value, $E(q)$ and $K(q)$ are complete elliptic integrals with modulus $q^{1 / 2}$. It is easy to see that at $q \rightarrow 0$ the function (14) is close to a sinusoid, while at $q \rightarrow 1$ it becomes a train of pulses close to solitons. Substituting (14) into (13) one obtains an equation describing time variation of $q$

$$
\frac{\mathrm{d} Y_{1}(q)}{\mathrm{d} t}=-2 \varepsilon Y_{1}(q)-4 \delta_{T} k^{2} Y_{2}(q)+\frac{2}{\pi} k \mu Y_{3}(q)-\frac{2}{\pi} v k^{3} Y_{4}(q),
$$

where (cf. [16]):

$$
\begin{aligned}
& Y_{1}(q)=\frac{K^{4}}{\pi^{2}}\left[\frac{4-2 q}{3} \frac{E}{K}-\frac{1-q}{3}-\frac{E^{2}}{K^{2}}\right] \\
& Y_{2}(q)=\frac{4 K^{6}}{15 \pi^{4}}\left[2\left(q^{2}-q+1\right) \frac{E}{K}-(1-q)(2-q)\right] \\
& Y_{3}(q)=K^{2}\left\langle\frac{\mathrm{d} z_{n}}{\mathrm{~d} \theta} f_{-\infty}^{\infty} \frac{\mathrm{d}^{2} z_{n}}{\mathrm{~d} \theta^{\prime 2}} \cdot \frac{\mathrm{d} \theta^{\prime}}{\theta-\theta^{\prime}}\right\rangle \\
& Y_{4}(q)=K^{4}\left\langle\frac{\mathrm{d} z_{n}}{\mathrm{~d} \theta} \int_{-\infty}^{\infty} \frac{\mathrm{d}^{4} z_{n}}{\mathrm{~d} \theta^{\prime 4}} \cdot \frac{\mathrm{d} \theta^{\prime}}{\theta-\theta^{\prime}}\right\rangle .
\end{aligned}
$$

This equation is generally rather complicated for analytical investigation. At $q \rightarrow 0$ (16) gives a quasi-sinusoidal wave with an amplitude growing or damping as an exponent defined by 
(9). In the other limiting case $q \rightarrow 1$ this gives an equation for the amplitude of the soliton, i.e. the wave of the type

$$
\eta(x, t)=A s \operatorname{sh}^{2} \frac{x-V_{s} t}{\Delta}
$$

where $V_{s}=V\left(1+\frac{\alpha A}{3}\right), \Delta=\sqrt{\frac{12 \beta}{\alpha A}}$. It has the form

$$
\begin{aligned}
\frac{\mathrm{d} A}{\mathrm{~d} t}= & -\frac{4}{3} \varepsilon A+\frac{2.92}{\pi} \mu\left(\frac{\alpha}{12 \beta}\right)^{1 / 2} \cdot A^{3 / 2}-\frac{16}{15} \frac{\alpha \delta_{T}}{12 \beta} A^{2} \\
& -\frac{5.11}{\pi} \nu\left(\frac{\alpha}{12 \beta}\right)^{3 / 2} \cdot A^{5 / 2}
\end{aligned}
$$

This equation also follows directly from (13) after substituting (18).

Analysis of Eq. (19) shows that besides a stable zero equilibrium state there are two other equilibrium values of $A$ : a stable one $\left(A_{\mathrm{st}}^{\prime}\right)$ and an unstable one $\left(A_{\mathrm{st}}^{\prime \prime}<A_{\mathrm{st}}^{\prime}\right)$. Thus, hard excitation conditions are realized in this system: decay of solitons with an initial amplitude $A_{0}$ below some threshold value $A_{\mathrm{st}}^{\prime \prime}$ and amplification of solitons with a sufficiently large amplitude $\left(A>A_{\mathrm{st}}^{\prime \prime}\right)$ up to the stationary value $A_{\mathrm{st}}^{\prime}$. The amplitude of solitons with $A_{0}>A_{\mathrm{st}}$ decreases down to the same value $A_{\mathrm{st}}^{\prime}$. Figure 6 shows time dependence of soliton amplitudes for different initial conditions. At $\varepsilon=0$ hard excitation is changed by the soft one $\left(A_{\mathrm{st}}^{\prime \prime}=0\right)$ and zero-amplitude state is unstable. Note, that in this case, provided that $A_{0} \ll A_{\mathrm{st}}^{\prime}$, explosive growth is characteristic of solitons at the initial stage:

$$
\frac{A}{A_{0}} \sim \frac{1}{\left(1-\frac{t}{t_{\text {expl }}}\right)^{2}}
$$

where $t_{\text {expl }}=\frac{2 \pi}{1.46 \mu} \sqrt{\frac{3 \beta}{\alpha A_{0}}}$. Subsequently explosive growth is restricted and solitons

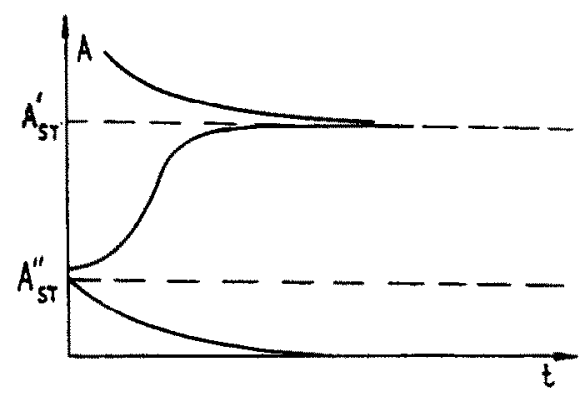

Fig. 6. Qualitative dependences of soliton amplitudes on time at different initial conditions.

approach a stationary stage $\left(A=A_{\mathrm{st}}^{\prime}\right.$ ) [formula (20) remains valid at $\varepsilon \neq 0$ if $A_{\mathrm{st}}^{\prime \prime} \ll A_{0} \ll A_{\mathrm{st}}^{\prime}$ ] The stationary soliton amplitude is shown in Fig. 7 as a function of the upper layer thickness $h$ for two values of $N$. Note, that at sufficiently large $h$ the wave height becomes of the same order as $h$, which seems to indicate breaking of such a strongly-non-linear wave before reaching its stationary value. On the other hand, there exist some minimal values of $h$ at which instability is still possible. Note, that the validity of Eq. (19) associated with smallness of $\hat{S}(\eta)$ becomes better as the soliton grows, in contrast to the cases of soliton damping.

In a more general case of cnoidal wave we have not succeeded in constructing analytical solutions of Eq. (12). However, if the amplification is sufficiently large, i.e. $q \rightarrow 1$, a cnoidal wave transforms to a periodic sequence of solitons, with (19) being valid for each of them. 


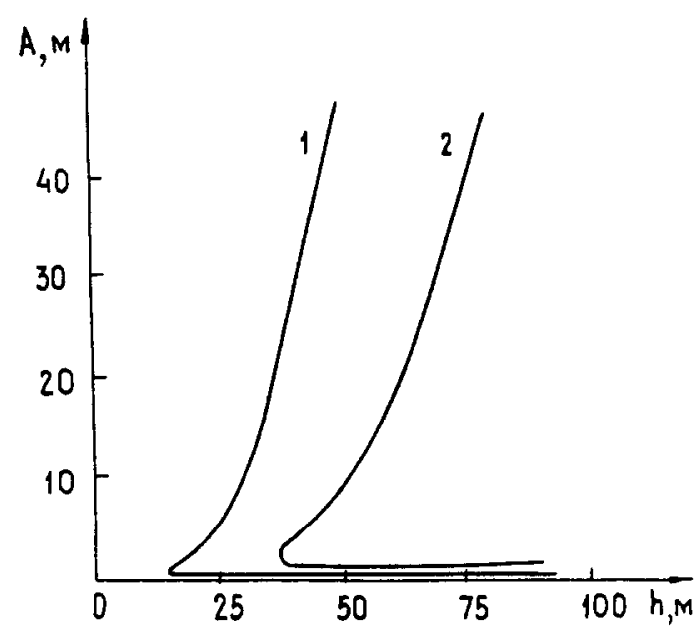

Fig. 7. Stationary soliton amplitudes $A_{\mathrm{st}}^{\prime}$ (the upper branches of the curves) and $A_{\mathrm{st}}^{\prime \prime}$ (the lower branches of the curves) as a function of the upper layer thickness $h$ for two values of $N: I-$ $N=10^{-3} \mathrm{~s}^{-1} ; 11-N=5 \times 10^{-4} \mathrm{~s}^{-1}$ (the other parameters are the same as in Fig. 4).

This theory enables one to obtain analytical results for a non-linear stage of hydrodynamic instability. At the same time it imposes a number of restrictions. In the first place, losses should not be too small, otherwise soliton growth results in amplitudes comparable with their length and non-linearity ceases to be weak. In the second place, at small flow velocities $\left(U<U_{c}\right)$ only waves with finite values of $K$ rather than long waves are unstable. In some cases the amplification band of such waves may be quite narrow, then adequate description of the wave process can be obtained by generalizing a non-linear Schrödinger equation with additional active and dissipative terms (cf. $[17,18]$ ). At sufficiently strong instability a stochastic non-linear process is possible in this case. This has been illustrated in $[17,18]$ as applied to a non-linear Schrödinger equation, as well as in [19] based on numerical computations of the equation whose structure resembles that of Eq. (12).

\section{DISCUSSION}

In conclusion let us summarize the results and discuss briefly possible related oceanographic effects.

In a model of a sharp pycnocline as an interface of two homogeneous fluids, at a finite depth of the moving upper layer the Kelvin-Helmholtz instability occurs only in a sufficiently short-wave region. For a stratified lower fluid long-wave radiation, instability occurs that is associated with NEW growth due to energy loss by radiation. The radiation instability band is separated from the $\mathrm{KH}$ one by a stable interval and at the same conditions $\left(U>U_{c}\right)$ it ranges from $k=0$ to $k=k_{M}<k_{\mathrm{KH}}$. Note, that although in the given model $\mathrm{KH}$ instability turns to be "stronger" than $R 1$, in real conditions this difference may diminish due to high-frequency losses (see, for example, [20] where dependence of KH instability on viscosity was studied) and, particularly, due to finiteness of the shear layer thickness. As follows from numerical computations [21], in stratified shear flows with finite pycnocline thickness the KH and RI increments may be of the same order. The spatial growth rate of perturbations for KI turns to be even larger owing to small group velocity of long NEW (see Fig. 3).

Note, that the NEW concept seems to have a rather wide area of applications. Thus, in several recent papers (see, for example, [22]) a special type of instability has been considered which is associated with resonant over-reflection of internal waves from a shear-flow layer in a fluid with $N=$ const. Although the approach used in these papers is different from ours, it may be easily shown that negative-energy waves occur in both cases. The main difference is that the existence of instability caused by resonant over-reflection requires the presence of a rigid boundary providing "feedback", while RI may develop at the density discontinuity sustaining the corresponding NEW mode.

Weak dispersion and instability effects in the long-wave region enable one to investigate a non-linear stage of RI within the frames of generalization of a $\mathrm{KdV}$ equation. Account of 
turbulent viscosity in the upper layer leads to saturation and onset of a stable stationary stage. Allowance for turbulent density diffusion near the pycnocline results in hard excitation for solitons when only solitary waves whose amplitudes are larger than the threshold one $A_{\mathrm{si}}^{\prime \prime}$ but smaller than $A_{s 1}^{\prime}$ are amplified.

Radiation instability is likely to occur in the ocean. A typical structure of the upper ocean layer is in good qualitative agreement with the one shown in Fig. 1. If $\Delta \rho / \rho=10^{-3}$, $N=5 \times 10^{-3} \mathrm{~s}^{-1}$ and $U / U_{c}=1.1, \mathrm{RI}$ is most effective at $h \simeq 10-50 \mathrm{~m}$ for the wavelengths $10^{2}-10^{3} \mathrm{~m}$ and the time scale $20-200 \mathrm{~min}$. The characteristic time of instability in this case is of the order of a day. It is not excluded that the processes described here are among the sources of short-period internal wave trains in the ocean.

\section{REFERENCES}

1. J. S. Turner, Buofancy Effects in Fluid. Cambridge University Press, Cambridgc (1973).

2. E. E. Gossard and W. H. Hooke, Waves in the Atmosphere. Elsevier, Amsterdam (1975).

3. P. C. Drazin and L. N. Howard, Hydrodynamic stability of parallel flow of inviscid fluid. Adv. appl. Mech. 9, 1 (1966).

4. T. B. Benjamin, The threefold classification of unstable disturbances in fiexible surfaces bounding inviscid flows. J. Fluid Mech. 16, 436 (1963).

5. M. V. Nezlin, Negative energy waves and anomalous Doppler effect. Uspekhi Fiz. Nauk 120, 481 (1976). (In Russian.)

6. R. A. Cairns, The role of negative energy waves in some instabilities of parallel flows. J. Fluid Mech.92, 1 (1979).

7. A. G. Voronovich and S. A. Rybak, Explosive instability of stratified flows. Dokl. Akad. Nauk SSSR 239, 1457 (1978) (In Russian).

8. A. D. Craik and J. A. Adam, "Explosive" resonant wave interactions in a three-layer fluid flow. J. Fluid Mech. 92 , 15 (1979).

9. L. A. Ostrovsky and Yu. A. Stepanyants, Non-linear stage of shear instability in a stratified fluid of finite depth. Izv. Akad. Nauk SSSR, Mekhanika Zhidk, i Gaza 4,63 (1982)(In Russian). [English translation in Fluid Dynamics 17, 540 (1983).]

10. L. A. Ostrovsky and L. Sh. Tsimring, Radiation instability of shearflows in a stratified fluid. Izv. Akad. Nauk SSSR, Fizika Atmosfery i Okeana 17,766 (1981)(In Russian). [English translation in Atmos. Ocean Phys. 17, 564 (1981). ]

11. L. D. Landau and E. M. Lifshitz, Fluid Mechanics. Pergamon Press, London (1959).

12. L. A. Ostrovsky and I. A. Soustova, The upper mixed layer of the ocean as an energy sink of internal waves. Okeanologiya 19 (6), 973 (1979) (In Russian). [English translation in Oceanology 19, 643 (1980).]

13. G. I. Barenblatt, Strong interaction of gravity waves and turbulence. Izv. Akad. Nauk SSSR, Fizika Atmosfery $i$ Okeana, 13 (1977) (In Russian).

14. L. A. Ostrovsky, Short-wave asymptotics for weak shock waves and solitons in mechanics. Int. J. Non-linear Mech. 11, 401 (1976).

15. V. I. Karpman, Non-linear Waves in Dispersive Media. Pergamon Press, Oxford (1978).

16. E. N. Pelinovsky, Non-linear ion-acoustic waves in inhomogeneous weakly absorbing plasma. $/ z v$. Vuzot, Radiofizika 14, 1281 (1971) (In Russian).

17. Y. Kuramoto and T. Yamada, Turbulent state in chemical reactions. Prog theor. Phys. 56, 679 (1976).

18. M. I. Rabinovich and A. L. Fabrikant, Stochastic self-modulation of waves in nonequilibrium media. $Z h$. Eks. Teor. Fiz. 77, 617 (1979) (In Russian).

19. Y. Kuramoto and T. Tsuzuki, Persistent propagation of concentration waves in dissipative media far from thermal equilibrium. Prog. theor. Phys. 55, 510 (1976).

20. N. G. Kikina, Viscosity effect on instability of tangential discontinuity in incompressible medium. Akust. $Z$ h. 13, 213 (1967) (In Russian).

21. D. C. Fritts, Simple stability limits for vertically propagating unstable modes in a tanh (z) velocity profile with a rigid lower boundary. J. atmos. Sci. 37. 1642 (1980).

22. R. H. T. Grimshaw, Resonant over-reflection of internal gravity waves from a thin shear layer. J. Fluid Mech. 109,349 (1981).

\section{Résumé:}

Cet article traite d'un nouveau mécanisme d'instabilité dans

des fluides stratifiés provoqué par un rayonnement d'onde

interne à partir d'une couche de cisaillement. Cette instabilité de rayonnement peut être expliquée en termes du concept de l'onde d'énergie négative. Ces ondes peuvent grossir à cause des pertes de rayonnement. On analyse la relation de dispersion et l'étape non linéaire du processus sur un modèle avec une discontinuité tangentielle de vitesse. La description non linéaire est basée sur une approximation d'onde longue conduisant à une équation d'évolution du type Korteweg - de Vries avec certains termes additionnels responsables de l'instabilité comme de la dissipation due à la turbulence. On obtient des solutions approchées de l'équation qui, en particulier, décrivent l'évolution des solitons y compris la croissance explosive et 1 'approche de l'état stationnaire. 
Zusammenf as sung:

Diese Arbeit behandelt einen neuen Mechanismus der Instabilitaet in geschichteten Fluessigkeiten, der durch die innere Ausbreitung einer Welle von einer Schubschicht verursacht wird. Dies Instabilitaet kann mit Hilfe des Begriffes von Wellen mit Negativenergic erklaert werden. Dies Wellen koennen durch Ausstrahlungsverluste anwachsen. Die Dispersionsbeziehung und der nichtlineare Abschnitt des Prozesses werden an Hand eines Models mit einer Diskontinuitaet der Tangentialgeschwindigkeit untersucht. Die nichtlineare Beschreibung basiert auf einer Annaeherung mit langen Wellen. Sie fuehrt zu einer Entwicklungsgleichung nach Korteweg und de Vries, die bestimmte fuer die Instabilitaet und die Turbulenzdissipation verantwortliche Glieder enthaelt. Die Gleichung Wird naeherungsweise geloest wodurch insbesondere die Entwicklung explosiven Anwachsens und die Annaeherung an den stationaeren Zustand beschrieben wird. 